\title{
INTERACTIONS OF WAVES WITH IDEALIZED HIGH-RELIEF BOTTOM ROUGHNESS
}

\author{
Xiao Yu, University of Florida, yuxiao03@ufl.edu \\ Johanna H. Rosman, University of North Carolina at Chapel Hill, jrosman@ unc.edu \\ James L. Hench, Duke University, jim.hench@duke.edu
}

\begin{abstract}
Interactions between waves and high-relief bottom roughness were investigated using Large Eddy Simulations of oscillatory flow over an infinite array of regularly spaced hemispheres. Simulation results were analyzed using a spatially- and phase-averaged momentum balance to provide insight into how flowtopography interactions affect wave-driven oscillating flows. Phase-averaging was applied first, and then spatial averaging was applied over volumes with horizontal length scales greater than the size of a single solid obstacle but fine enough in the vertical direction that the vertical structure of the dynamics was resolved. Spatial averaging of the momentum equation results in terms that represent drag and inertial forces, and a dispersive stress term that represents a vertical momentum flux induced by the spatial heterogeneity of the phase-averaged flow. These new terms require parameterization in coastal ocean wave and circulation models.
\end{abstract}

Flow dynamics strongly depends on KeuleganCarpenter number $K C=U_{0} T / D$, where $U_{0}$ is the amplitude of the wave velocity, $T$ is the wave period and $D$ is the diameter of a single hemisphere. At low $K C$ $(1<K C<10)$, there is no strong flow separation and instabilities formed near flow reversal contributed significantly to turbulence generation. The inertial force dominated flow dynamics, form drag was small, and both Reynolds and dispersive stresses were negligible. At higher $K C(10<K C<20)$, strong flow separation occurred and turbulence was primarily generated in the wake. Both drag and inertial forces were important in the momentum budget. The dispersive stress was the main mechanism for vertical momentum transfer, although the stress gradient term was small compared with drag and inertial force terms. The drag coefficient for each hemisphere increased as $K C$ increased due to the increasing strength of flow separation, and appeared to approach a constant value at high $K C$. The added mass coefficient for each hemisphere decreased as $K C$ increased. Furthermore, the drag coefficient was insensitive to the spacing $(S)$ between adjacent hemispheres and flow sheltering was negligible; however, the added mass coefficient decreased as $S$ decreased, because the amount of fluid affected by a single hemisphere was limited by $S$.

Most models that are commonly used to estimate the friction factor $f_{w}$ were developed for $\zeta / k_{s}>1$, when the wave boundary layer thickness $(\zeta)$ is large compared with roughness element height $\left(k_{s}\right)$, shear stress at the top of the roughness layer is similar to form drag per unit area exerted by the bed, and the inertial force is small compared with form drag. The approximate region in which these assumptions are valid are indicated in Fig. 1 as the non-shaded region. None of these assumptions are true for $\zeta / k_{s}$ of unity or smaller. In this parameter range, models for the friction factor must explicitly account for form drag and inertial forces. It is expected that friction factors for $\zeta / k_{s}<1$ depend strongly on the three-dimensional structure of the bottom.

Friction factors were calculated from simulation results based on the drag force $\left(f_{w, d}\right)$ alone and the total force $\left(f_{w, t}\right)$. As $K C$ increased, $f_{w, d}$ increased due to the increasing strength of flow separation and approached previously proposed curves at high $K C$. However, $f_{w, t}$ decreased monotonically as $K C$ increased due to the decreasing magnitude of the inertial force, and matched previously proposed empirical/semi-empirical curves reasonably well for all $K C$. These results highlight the importance of distinguishing the total force on the bottom, the drag force that removes the energy from the flow, and the shear stress above the obstacle layer, which were very different for the parameter range in this study.

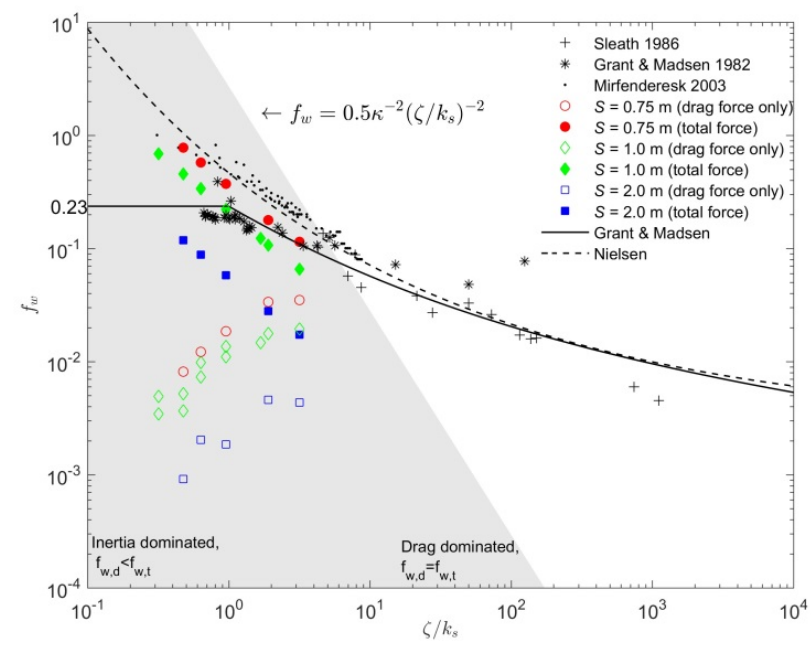

Figure 1 Friction factor $f_{w}$ versus $\zeta / k_{s}$. Solid line represents the theoretical solution by Grant \& Madsen (1982). Dashed curve is the empirical formula by Nielsen (1992).

\section{REFERENCES}

Grant and Madsen (1982): Movable bed roughness in unsteady oscillatory flow. J. Geophys. Res. (Oceans), 87(C1), 469-481.

Nielsen (1992): Coastal bottom boundary layers and sediment transport. World Scientific.

Sleath (1986): Turbulent oscillatory flow over rough beds, J. Fluid Mech., 182, 369-409.

Mirfenderesk (2003): Direct measurements of the bottom friction factor beneath surface gravity waves. Applied Ocean Res., 25(5), 269-287. 